\title{
Surface Micromachined Pressure Sensors for Medical Applications
}

Trieu, Hoc Khiem

Email: trieu@tu-harburg.de

Fraunhofer IMS, Finkenstr. 61, 47057 Duisburg,

TU Hamburg-Harburg, Institute of Micro System Technology, Eißendorfer Straße 42, 21073 Hamburg

\begin{abstract}
For a variety of diseases such like hypertension, hydrocephalus, or glaucoma pressure is a relevant physiological parameter. In novel treatment concepts using long term monitoring or telemedicine platforms implantable micro pressure sensor transponders gain increasing importance due to their minimal invasiveness, their capability to collect long term data and fully automated operation. Monolithic integrated capacitive pressure sensors provide some unique characteristics such as extreme miniaturization, on-chip electronics and low-power operation. These features are essential for the development of implantable devices. The basis of surface micromachined pressure sensors as well as their potential are presented in this paper with the examples of some selected medical applications.
\end{abstract}

\section{Keywords}

capacitive pressure sensor, surface micromachining, transponder, micro implant, medical device.

\section{INTRODUCTION}

Pressure is physiologically a very interesting parameter in medical diagnostics. From blood pressure for patients suffering from hypertension to cranial pressure in hydrocephalus, intraocular pressure in glaucoma or bladder pressure in incontinence as some examples, it covers a broad range of relevance of different diseases. Facing new challenges in health care as a result of demographic change and an accompanied increase in life expectancy as well as the high incidence of widespread diseases in industrialized countries, new techniques for early diagnosis in prevention as well as novel concepts for long term monitoring using micro implants and telemedicine platforms are introduced.

For the development of micro implants with the capability of telemetric operation two requirements are essential for the technology in use: miniaturization and low power consumption. The presented technology of surface micromachined capacitive pressure sensors has successfully demonstrated to fulfil these characteristics in various research and product development projects. For each application the packaging and implantation technique has to be adapted in order to meet the physiological condition.

\section{INTEGRATED PRESSURE SENSOR TRANSPONDER}

\section{Surface Micromachined Pressure Sensor}
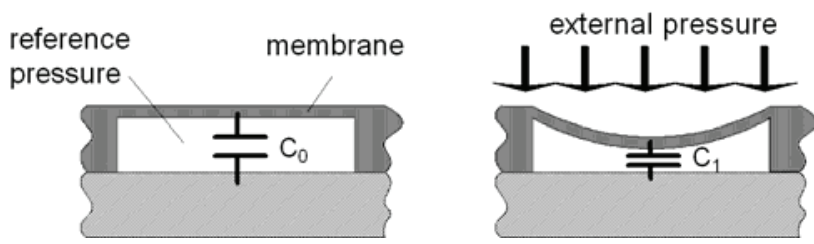

Fig. 1 Principle of a capacitive pressure transducer.

Micromachined capacitive pressure sensor is a mature technology that is well-known [1]. Thin diaphragms which deflect under pressure are appropriate basic structures for the detection hereof. A deflected plate shows specific stress and strain states proportional to the deflection and hence proportional to the pressure. For strain based sensors the distance change between the deflected

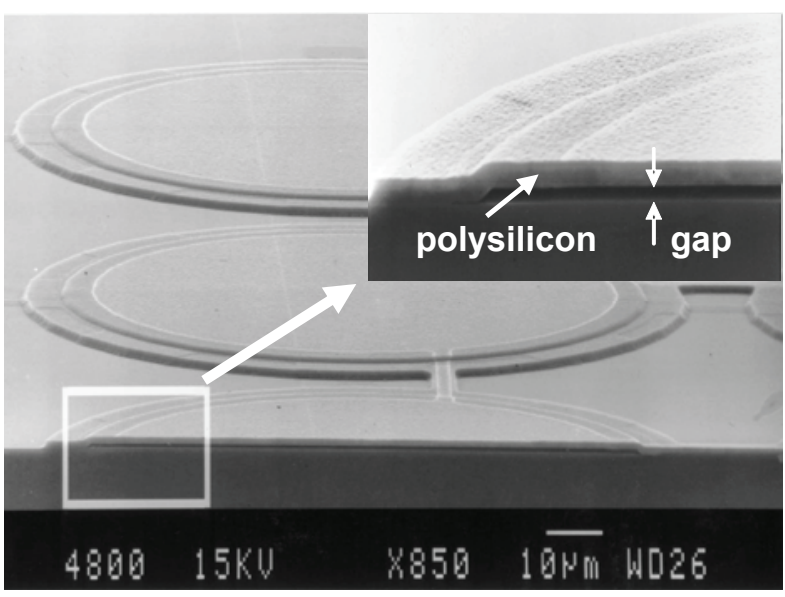

Fig. 2 Micro plate capacitor for pressure sensing. 
plate and another fixed plate can be used to detect a change in the capacitance, provided both plates are conducting, as illustrated in figure 1. Known for its ideal elastic behaviour silicon is an excellent material for the creation of a diaphragm.

A simple way to fabricate a micro plate capacitor is to apply surface micromachining. Figure 2 shows SEM images of a fabricated pressure sensor array. The basic steps of the process flow used for the fabrication of the device are depicted in figure 3 . The bottom electrode is an implanted $n+$-area in a p-type

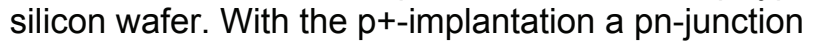
is formed for electrical isolation of the $n+$-electrode from the substrate. A dielectric layer is deposited to electrically isolate the bottom electrode from the later formed top plate. The pattern of the spacer oxide and channel oxide defines the cavity between the two electrodes. Now the pressure sensitive polysilicon diaphragm as the top electrode is deposited and patterned leaving the etch channel accessible to the hydrofluoric acid. The etchant removes selectively the oxide resulting in a cavity which then is sealed under vacuum condition, resulting in an absolute pressure sensor cell. After metallization and passivation the passivation layer is locally removed from the polysilicon diaphragm of the pressure sensitive elements.

The pressure range is design controlled by adjusting the diameter of the sensor diaphragm, keeping the thickness to be fixed. This allows the fabrication of various sensors for low (1 bar max.) up to high (350 bar max.) pressure ranges on a same wafer or even on a same device. For atmospheric pressure the diaphragm diameter is about $100 \mu \mathrm{m}$. A close up view of a sensor cell reveals the extreme aspect ratio of the diaphragm diameter and the cavity height considering that the diameter is in the range of some tens up to a hundred micrometer. The small cavity height $(<1 \mu \mathrm{m})$ limits the deflection of the diaphragm automatically, once the top plate touches the bulky bottom substrate.

In order to readout the small capacitance of the pressure sensor cell only very small current is needed to charge or discharge the capacitor. Combining this with CMOS circuits allows the realization of low power consuming systems.

Since the surface micromachining technique is a planar technology similar to a CMOS process the pressure sensor process module can be integrated as depicted in figure 4. A co-integration of pressure sensor and CMOS process has been implemented. A high voltage option and EEPROMs are also available. Figure 5 shows an example of a tiny integrated pressure sensor chip.

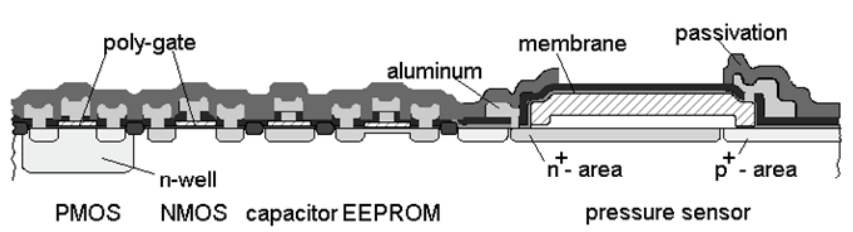

Fig. 4 Monolithic integration of sensor and CMOS.

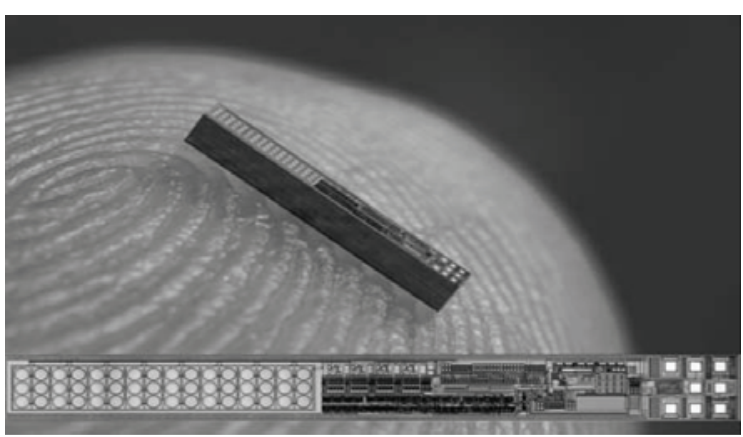

Fig. 5 Integrated pressure sensor on finger tip. 


\section{Micro Pressure Sensor Transponder}

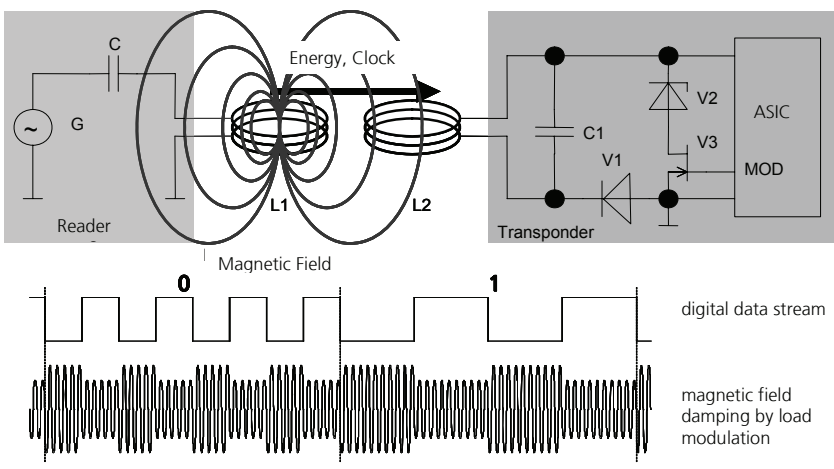

Fig. 6 Principle of inductive coupling for energy transfer. Field damping is additionally applied for data transfer.
Combining the low-power integrated pressure sensor with the technology of inductive coupling micro pressure sensor transponders are realized. The principle of inductive coupling for energy and data transfer is illustrated in figure 6 . As an implantable device the micro implant transponder consists of an integrated capacitive pressure sensor chip and a coil including some discrete electronic components for the transmission of power and data. The on-chip electronics contains an RFinterface for the wireless communication. A magnetic field with a frequency of $133 \mathrm{kHz}$ or $13.5 \mathrm{MHz}$ is generated by an external coil that is connected to a handheld reader. The field transfers power to the second coil of the

pressure sensor transponder located inside the implant by inductive coupling. Apart from the power, the transponder extracts its clock from the frequency of the magnetic field. The digitized pressure values of the sensor are Manchester and CRC coded. Transfer of the values is done by load modulation that causes damping of the magnetic field. The field damping is evaluated by the reader. If the CRC of the transferred data is valid, pressure values are processed further.

\section{MEDICAL APPLICATIONS}

Based on the described micro pressure sensor transponder technology various research and product developments have been conducted. Some examples of medical applications are presented in the following demonstrating the versatility of the technology.

\section{Intraocular Pressure Sensor System for Monitoring of Glaucoma}

For patients suffering from glaucoma the intraocular pressure has to be regularly monitored. An implanted pressure sensor system [2] as depicted in figure 7 allows a direct measurement of the intraocular pressure without being affected by individual geometry of the eyes as in case of indirect external measurements.
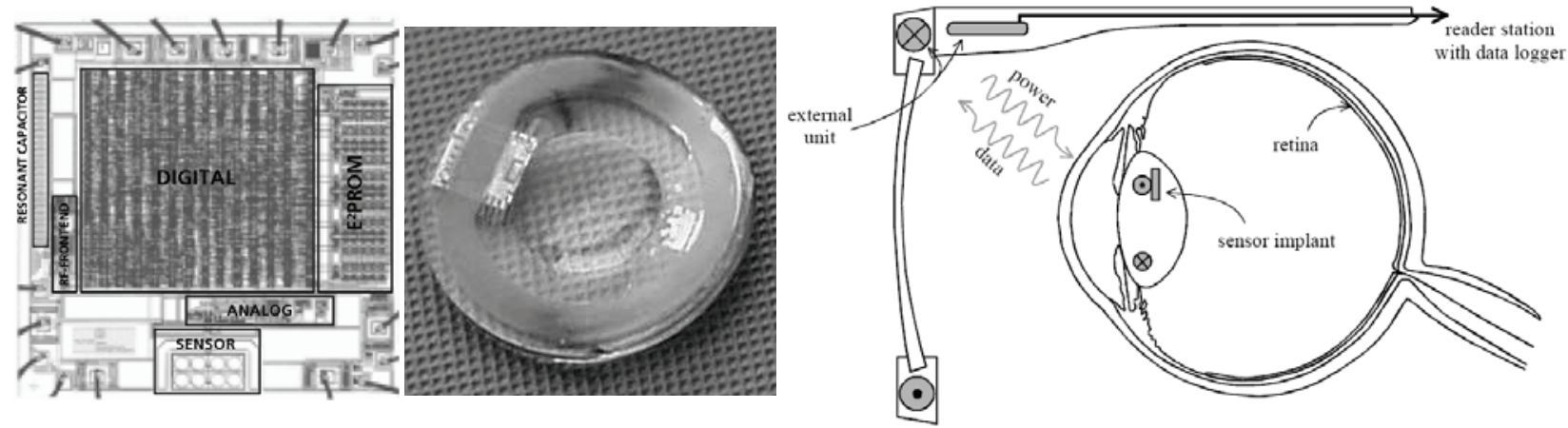

Fig. 7 Intraocular Pressure Sensor System. Micrograph of the integrated pressure sensor (left), sensor chip connected to micro coil and encapsulated in intraocular lens (middle), architecture of the intraocular pressure sensor system (right).

The intraocular pressure sensor system consists of two major components: An external reader unit and the sensor implant. The reader antenna connected to a reader station with a data logger is placed in front of the eye. The sensor implant consists of a $2.6 \times 2.6 \mathrm{~mm}^{2}$ integrated pressure sensor chip which is connected to a micro coil on a polyimide foil by flip-chip bonding technique. This setup is finally encapsulated in an intraocular lens. The implant is placed inside the lens capsule. Once the external unit is active transmitting power the implant extracts the power and clock from the alternating magnetic field $(13.5 \mathrm{MHz})$. The sensor measures pressure and temperature. The values are digitized, Manchester and $\mathrm{CRC}$ coded. These data are transmitted back to the external reader via field damping. 


\section{Pressure Sensor Capsule for Abdominal Aorta Aneurysm Control}

For the treatment of abdominal aortic aneurysms (AAA) keyhole surgery has been established using foldable stent grafts. One potential problem of this method is the occurrence of leakages. In a research project a pressure sensor capsule, as shown in figure 8 , was developed for the monitoring of leakages [3]. The capsule consists of a pressure sensor ASIC, a receiver coil, 2 zener diodes, and 2 capacitors. The components are assembled on a polyimide micro flex-tape, then moulded into a biocompatible silicone forming the shape of the capsule, and finally coated with a Parylene-C layer. Due to the required longer transmission distance a carrier frequency of $133 \mathrm{kHz}$ was chosen. For this lower frequency, comparing to the intraocular system operating at $13.5 \mathrm{MHz}$, the passive components needed for the tunable resonant circuit have to be realized as off-chip discrete devices. For this setup a transmission range of up to $120 \mathrm{~mm}$ was achieved. The function of the implant was tested in an in-vitro test environment using a blood circulation model. The result shown in figure 8 (right) depicts a measurement with an artificial induced leakage at a systolic/diastolic setting of 70/130 $\mathrm{mmHg}$.

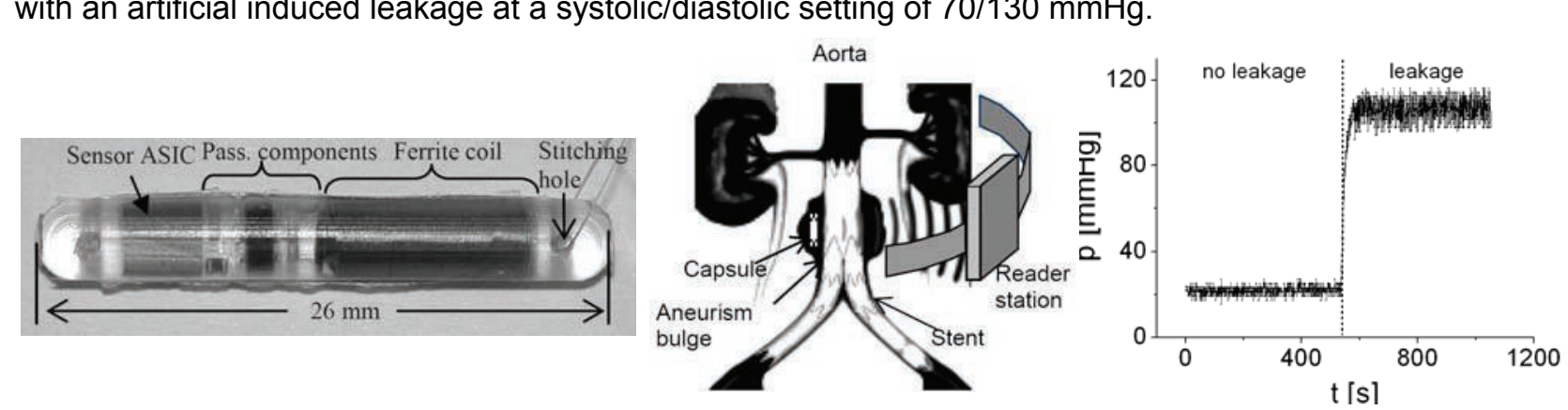

Fig. 8 Pressure sensor capsule for AAA control. Micrograph of the sensor capsule (left), scheme of the sensor system (middle), result from measurement in a blood circulation model (right).

\section{Implantable Blood Pressure Sensor for Hypertonic Patients}

Hypertension is a widespread disease. Approximately 10 million people suffer from hypertension in Germany. About $10 \%$ of these patients can hardly be stabilized on drugs and for another $10 \%$ of this group a long term monitoring is advisable. An implantable blood pressure sensor for $24 / 7$ monitoring was developed [4], [5], [6], [7]. The implant consists of a tiny sensor chip integrated at the tip of a catheter ( $\varnothing$ $1.1 \mathrm{~mm}$ ) and a telemetric unit (figure 9). The sensor-tip is placed into the femoral artery while the telemetric unit is implanted subcutaneously (figure 10). The implant is supplied with power wirelessly via inductive coupling from an external reader station. Data is readout from the external station with approx. $30 \mathrm{~Hz}$ and an overall accuracy of $\pm 2.5 \mathrm{mmHg}$ is achieved. Figure 11 shows the transient blood pressure curve from the femoral artery of an anaesthetized sheep, recorded telemetrically with a distance between the implanted transponder unit and the extracorporal reader unit of $10 \mathrm{~cm}$. The rather low levels in blood pressure from $30 \mathrm{mmHg}$ to $80 \mathrm{mmHg}$ are suspected to be matter of the anaesthesia of the sheep.

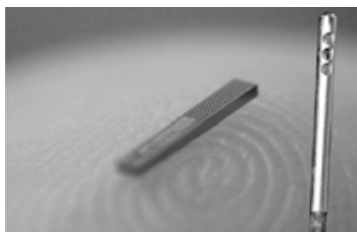

sensor tip

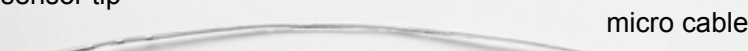

transponder unit

Fig. 9 Blood pressure sensor implant. Micrograph of the sensor tip and the tiny sensor chip (left), implant consists of sensor tip, micro cable and transponder unit (right).

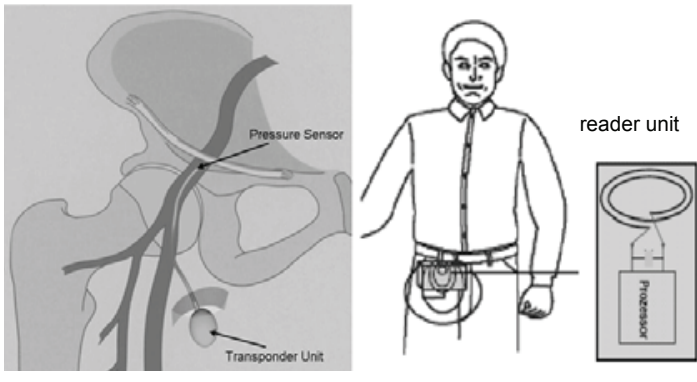

Fig. 10 Illustration of the monitoring system.

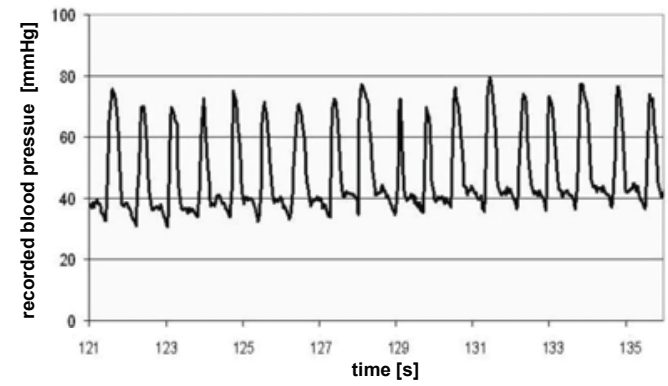

Fig. 11 Recorded data from an anaesthetized sheep. 


\section{Pulmonary Artery Pressure Monitoring in Heart Failure Patients}

Chronic heart failure is the cardiac disease with the highest increase rate. The prevalence increased by $165 \%$ in the period from 1979 to 2000 . The increase rate of death is $+148 \%$. It is the most common reason for hospital admission in Germany and one of the most cost-intensive chronic diseases. A precise and continuous check of the haemodynamic situation will enable an optimization of the medical therapy. It is expected that an early recognition of an increase of the pulmonary arterial pressure can serve as an early warning system for the physician in order to optimize the medicinal intervention, hence preventing a hospitalization (figure 12). In the project COMPASS the integrated pressure sensor technology is combined with a home monitoring platform in order to implement the afore-mentioned telemedicine scenario [8], [9]. As shown in figure 13 the COMPASS system consists of an integrated pressure sensor module connected to a subcutaneous implanted electronics / telemetric unit via a sensor cable, and a home monitoring station which transfers data by mobile communication via a service center to the attending physician. The sensor module contains besides the integrated pressure sensor an additional interface chip amplifying the signal gained from the sensor hence enables the signal transmission over the long distance between sensor and telemetric unit.

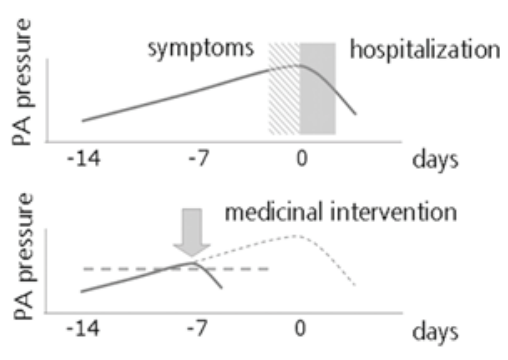

Fig. 12 Increase of pulmonary arterial pressure as an early warning prior to decompensation.

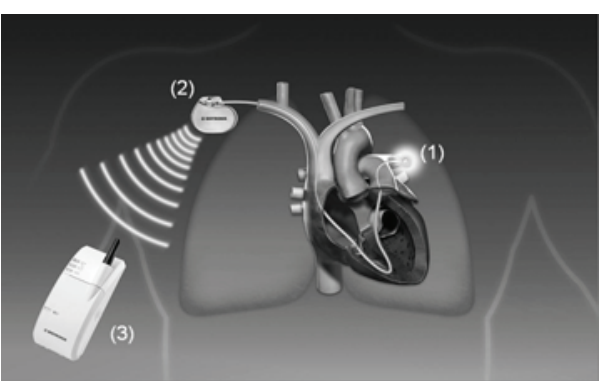

Fig. 13 The COMPASS system consists of micro pressure sensor (1), electronics (2), and reader (3).

\section{Intra Oral Tactile Sensing for Triggering a Pacemaker in the Treatment of Dysphagia}

Dysphagia or the difficulty in swallowing affects several ten thousands people in Germany each year. Often the swallowing act cannot be initiated due to a neural disease. A natural indicator for the start of the swallowing act is the characteristic movement of the tongue against the hard palate. In a feasibility study carried out in cooperation with the Research group MITI at the Klinikum r.d. Isar this movement was detected reliably by a telemetric pressure sensor that was fixed to the hard palate [10]. At the initial phase of swallowing the tongue moves against the hard palate to force the food towards the pharynx.

Consequently, if it would be possible to derive advantage from the tongue's characteristic movement by detecting the contact pressure of the tongue with the hard palate, a trigger can be use to initiate an electrical stimulation of the muscles in the throat. In this study the swallowing act was visualized by highspeed fluoroscopy and recorded pressure values were evaluated as shown in figure 14 . When the oral cavity is filled with contrast agent the pressure rises to 219 mbar. At the moment the test person intends to swallow his tongue presses automatically against the hard palate and thus the contrast agent towards the pharynx. This causes a strong increase of the pressure to 545 mbar indicating that swallowing starts immediately. Finally the contrast agent flows down the throat, the oral cavity empties and the pressure reading decreases to 92 mbar. These first investigations give very promising results and show that the approach to trigger swallowing by the signal of a pressure sensor that is caused by the natural movement of the patient's tongue is feasible. This gives future prospects to improve the treatment of dysphagia and to allow patients to continue eating in a natural way.
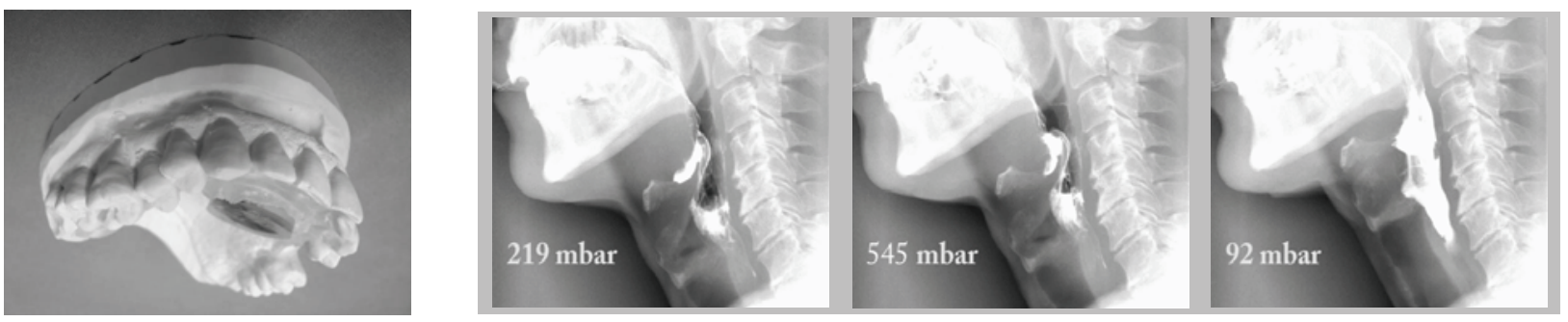

Fig. 14 In silicone encapsulated telemetric pressure sensor fixed to an imprint of the hard palate (left). Angiographs of the test person's oral cavity and throat at different phases of the swallowing act with the pressure values indicated (right). 


\section{SUMMARY}

The presented applications demonstrate the appropriateness and the versatility of surface micromachined capacitive pressure sensors for the development of micro implants. The monolithic integration of electronics and sensing elements allows the realization of extremely miniaturized systems. Low-noise sensor signal conditioning and a robust digital wireless data transmission are ensured by on-chip mixed signal electronics. The combination of CMOS circuitry and capacitive pressure sensing principle enables the low-power operation of the micro implants and consequently their use as passive transponders without the need of an implanted battery. Appropriate packaging and implantation techniques have been developed. They are prerequisite for a penetration of the technology in the various medical fields and will help to exploit further applications. The fabrication of the micro sensors using a qualified process in a fully automated CMOS production environment makes the technology suitable for high-volume cost-effective applications.

\section{ACKNOWLEDGEMENTS}

Parts of the results presented in this paper were achieved in the course of projects granted by the Federal Ministry for Education and Research (BMBF): HYPER-IMS (grant no 16SV2133), COMPASS (grant no 16SV3693).

\section{REFERENCES}

[1] Trieu, H.K.: Surface micromachined pressure sensor technologies. Proceedings Sensors Expo \& Conference Fall 2002, Boston, MA, September 23-26, 2002, pp. 111-122.

[2] Stangel, K.; Kolnsberg, S.; Hammerschmidt, D.; Hosticka, B.J.; Trieu, H.K.; Mokwa, W.: A programmable intraocular CMOS pressure sensor system implant. IEEE journal of solid-state circuits 36 (2001), Nr.7, pp. 1094-1100. ISSN 0018-9200.

[3] Schlierf, R.; Görtz, M.; Schmitz-Rode, T.; Mokwa, W.; Schnakenberg, U.; Trieu, H.K.: Pressure sensor capsule to control the treatment of abdominal aorta aneurisms. 13th International Conference on Solid-State Sensors, Actuators and Microsystems, Seoul, Korea, June 2005.

[4] Fassbender, H.; Urban, U.; Görtz, M.; Göttsche, T.; Trieu, H.K.; Mokwa, W.; Schmitz-Rode, T.: Fully implantable blood pressure sensor for hypertonic patients. THE SEVENTH IEEE CONFERENCE ON SENSORS (IEEE Sensors 2008) October, 26.-29. Lecce (ITALY)

[5] Trieu, H.K.; Göttsche, T.; Osypka, P.; Görtz, M.: Micro implants in medical technology with wireless data and power transmission. GMM-Workshop Energieautarke Sensorik 12.-13. November 2008. GMM-Fachbericht 58. ISBN 978-3-8007-3146-6.

[6] Göttsche, T.; Gräfe, M.; Osypka, P.; Görtz, M.; Trieu, H.K.; Fassbender, H.; Mokwa, W.; Urban, U.; Schmitz-Rode, T.; Bender, B.; Glocker, R.; Fähnle, M.: HYPER-IMS: A Fully Implantable Blood Pressure Sensor for Hypertonic Patients. SENSOR+TEST Kongress 2009; 26.05.2009; Nürnberg.

[7] Urban, U.; Fassbender, H.; Fürst, P.; Göttsche, T.; Bender, B.; Becker, R.; Mokwa, W.; Trieu, H.K.; Osypka, P.; Glocker, R.; Steinseifer, U.; Schmitz-Rode, T.: Fully implantable blood pressure system: implantation experiences. World Congress on Medical Physics and Biomedical Engineering 2009. Munich, Germany, September 7 -12, 2009.

[8] Müntjes, J.; Meine, S.; Flach, E.; Görtz, M.; Schmitz-Rode, T.; Trieu, H.K.; Mokwa, W.: Monitoring intravascular pressure with a pulmonary artery pressure sensor system - assembly aspects. Smart Systems Integration 2010. Como, Italy, March 23 - 24, 2010.

[9] Urbaszek, A.; Trieu, H.K.; Traulsen, Tim; Mokwa, W.; Schmitz-Rode, T.: Development of an Implantable Pressure Sensor for Continuous Pulmonary Artery Pressure Monitoring in Heart Failure Patients. BMT 2010, Rostok, October 5 - 8, 2010.

[10] Betz, W.; Görtz, M.; Feussner, H.; Schneider, A.; Knödgen, F.; Trieu, H.K.: Feasibility study of an integrated pressure sensor transponder for triggering of a pacemaker in the treatment of dysphagia. BMT 2010, Rostok, October 5 - 8, 2010. 